23 IMPROVING THE MANAGEMENT OF DENTAL TREATMENT FOR THE HOMELESS COMMUNITY WITH THE USE OF MOBILE DENTISTRY UNITS

Adam Tremlett. University of Exeter, UK

10.1136/leader-2019-FMLM.23

The chaos of homelessness and a high prevalence of previous trauma Results in self-neglect and therefore poor dental hygiene. It was found $99 \%$ of homeless people require dental treatment, however less than half completed their treatment plan. Reasons for this include dental anxiety and accessibility barriers, such as opening times and geographical inaccessibility. The lack of leadership and management focused on providing specific and suitable dental services for the homeless community leaves them with services that are not accessible.

Leading the way as a possible solution is a mobile dentistry unit (MDU) which would provide suitable dental care to the homeless population. It would be flexible, in location and time, and work collaboratively with an already established network of homeless service providers. In order to measure the success of this service it would be reviewed in areas including patient attendance, completion of treatment plans and patient satisfaction.

Although the initial financial cost would be high, $£ 200,000$, it would decrease the number of preventable hospital attendances and missed national health service (NHS) dental appointments. $55 \%$ of the homeless community miss their annual NHS dental appointments, and 30\% attend hospital with dental complaints. Based on the 580 people registered at Exeter's specialised homeless healthcare general practice surgery, The Clock Tower Surgery, Exeter, this would save $£ 65,000$ per year. Therefore, this would result in a long-term financial benefit after four years. There is also an incalculable benefit of pain relief, improved self-esteem and improved oral hygiene for the patients treated.

In conclusion, successful management and leadership of MDUs would tackle the need for dental services suitable for the homeless community. An increase in oral health can increase self-esteem and help gain a sense of control, which in turn can support the process of people getting their lives back on track.

\section{INFORMED CONSENT IN OBSTETRICS: A SURVEY OF PREGNANT WOMEN TO SET A NEW STANDARD IN INFORMED CONSENT FOR EMERGENCY OBSTETRIC INTERVENTIONS}

Tracey Sturgeon*, Huma Ayaz, Kirsty McCrorie, Kate Stewart. NHS Highland, Department of Obstetrics and Gynaecology, Raigmore Hospital, Inverness, UK (all authors)

\subsection{6/leader-2019-FMLM.24}

Respect for autonomy supports the rights of women to make their own decisions about care as laid out by the Supreme Court ruling on Montgomery (2015).Consent for emergency procedures in obstetrics presents a significant challenge.Consent obtained when a woman is exhausted, influenced by endogenous or exogenous chemicals or in fear of her unborn child's safety cannot be considered to be informed. An opportunistic survey of pregnant women in
Highland region was conducted over 6 weeks in community and secondary care antenatal clinics.Primary objective-determine women's current understanding of emergency obstetric interventions in labour to guide our work in achieving informed consent.Secondary objective-compare regional and Scottish national delivery data to allow realistic counseling of women regarding possibility of such interventions. Results were analysed and comments qualitatively explored.Labour and delivery expectations of survey participants were compared to regional and Scottish national delivery data (2018). We found that many women were uncertain regarding possibility of intervention. Both prim and parous women requested more information; some specifically asked for upto-date statistics. Regional and Scottish national delivery intervention rates were comparable.Current intervention rates (by regional and Scottish national data) are significantly higher than expected. Our data is in keeping with Scottish data so this is likely an issue in other regions too. Our survey showed pregnant women may not have realistic expectations of delivery outcomes. Pregnant women need information based on regional and national data to foster realistic expectations of labour or delivery; empowering decision-making and ensuring peri-partum emergency consent is still informed consent. A multi-disciplinary approach to a novel means of obtaining informed consent will allow NHS Highland to lead the way in implementing change to improve the care of our pregnant women.

\section{THE DARK OR LIGHT SIDE? INSIGHTS FROM THE GP- CONSULTANT EXCHANGE PROGRAMME}

${ }^{1,2,3}$ Pritti Aggarwal, ${ }^{4,5}$ Adam Fraser, ${ }^{6,7,8}$ Sally Ross. ${ }^{1}$ Southampton City Clinical Commissioning Group Board Member, UK; ${ }^{2}$ Primary Medical Care Module Lead, University of Southampton, UK; ${ }^{3}$ GP Partner, Living Well Partnership, Southampton, UK; ${ }^{4}$ GP Partner, Bridges Medical Practice, Weymouth, UK; ${ }^{5}$ Dorset Programme Director, Bournemouth University, UK; ${ }^{6}$ NHSE GP Clinical Advisor, UK; ${ }^{7}$ GP Tutor and Appraiser for Wessex, UK; ${ }^{8}$ Sessional GP UK

\subsection{6/leader-2019-FMLM.25}

Introduction and implementation Collaborative working across primary and secondary care is crucial to providing quality care. In this GP-Consultant Liaison scheme, 200 Consultants and GPs were strategically paired to enhance working relationships. Pairs hosted and visited each other's workplace. Our aim was to improve professional understanding, foster deeper partnership, ignite opportunities for innovation and quality improvement (QI) with co-owned local solutions. Submitted anonymous reflections were analysed for common themes. Celebrations of the shared learning took place regionally with affiliated NHS organisations. The emphasis was on compassionate leadership and next steps.

Impact Feedback obtained from 71(37.5\%) participants was scaled from 1 (least likely) to 6 (most likely). In breaking barriers, individuals supported a regular primary-secondary care forum; weighted average score of 5.25, found the scheme useful (4.59), likely to take part again (4.83), consider new ways of working consequent to building better relationships (3.85). We observed production of leaflets on psychological support for patients in hospital consequent to insight obtained from primary care, sharing directories of primary care secretary contacts and restructuring of outpatient clinic letters to state 'For Information Only' or 'GP Action Required' resulting in 